\title{
UNIVERSAL COVERS OF TOPOLOGICAL MODULES AND A MONODROMY PRINCIPLE
}

\author{
OSMAN MUCUK AND MEHMET ÖZDEMIR
}

\begin{abstract}
Let $R$ be a simply connected topological ring and $M$ be a topological left $R$-module in which the underling topology is path connected and has a universal cover. In this paper, we prove that a simply connected cover of $M$ admits the structure of a topological left $R$-module, and prove a Monodromy Principle, that a local morphism on $M$ of topological left $R$-modules extends to a morphism of topological left $R$-modules.
\end{abstract}

\section{Introduction}

Let $G$ be a connected topological group. It is a well known result that (see for example Chevalley's book [4]) if $p: \widetilde{G} \rightarrow G$ is a covering map such that $\widetilde{G}$ is a simply connected topological space, then $\widetilde{G}$ becomes a topological group such that $p$ is a morphism of topological groups.

The problem of universal covers of non-connected topological groups was first studied by Taylor in [10]. He proved that a topological group $G$ determines an obstruction class $k_{G}$ in $H^{3}\left(\pi_{0}(G), \pi_{1}(G, 0)\right)$, and that the vanishing of $k_{G}$ is a necessary and sufficient condition for the lifting of the group structure to a universal cover. In [6] an analogous algebraic result is given in terms of crossed modules and group objects in the category of groupoids (see also [1]) for a revised version, which generalises these results and shows the relation with the theory of obstructions to extension for groups).

The lifting problem of the ring structure of a connected topological ring to a simply connected was recently studied in [7] using the term of ring object in the category of groupoids.

The main object of this paper is to give an analogue of this work for topological $R$-modules rather than topological rings.

\section{Covering Maps}

We assume the usual theory of covering maps. All spaces $X$ are assumed to be locally path connected and semi locally 1-connected, so that each path component of $X$ admits a simply connected cover.

Received April 12, 2000; revised November 26, 2002.

2000 Mathematics Subject Classification. 22A05, 55M99, 55R15.

Key words and phrases. Universal cover, topological module, monodromy principle. 
Recall that a covering map $p: \widetilde{X} \rightarrow X$ of connected spaces is called universal if it covers every cover of $X$ in the sense that if $q: \widetilde{Y} \rightarrow X$ is another cover of $X$ then there exists a map $r: \widetilde{X} \rightarrow \widetilde{Y}$ such that $p=q r$ (hence $r$ becomes a cover). A covering map $p: \widetilde{X} \rightarrow X$ is called simply connected if $\widetilde{X}$ is simply connected. Note that a simply connected cover is a universal cover.

Definition 1.1. We call a subset $V$ of $X$ liftable if it is open, path connected and $V$ lifts to each cover of $X$, that is, if $p: \widetilde{X} \rightarrow X$ is a covering map, $i: V \rightarrow X$ is the inclusion map, and $\widetilde{x} \in \widetilde{X}$ satisfies $p(\widetilde{x})=x \in V$, then there exists a map (necessarily unique) $\hat{\imath}: V \rightarrow \widetilde{X}$ such that $p \hat{\imath}=i$ and $\hat{\imath}(x)=\widetilde{x}$.

It is easy to see that $V$ is liftable if and only if it is open, path connected and for all $x \in V, i_{\star} \pi_{1}(V, x)=\{0\}$ where $\pi_{1}(V, x)$ is the fundamental group of $V$ at the base point $x, i_{\star}$ is the map induced by the inclusion map $i: V \rightarrow X$ and $\{0\}$ is the identity element of the fundamental group $\pi_{1}(X, x)$. Remark that if $X$ is a semi locally simply connected topological space, then each point $x \in X$ has a liftable neighbourhood.

So if $X$ is a semilocally simply connected topological space then each $x \in X$ has a liftable neighbourhood.

Lemma 1.2.([9]) Let $X$ be a connected topological space and $p: \widetilde{X} \rightarrow X$ a covering map. Suppose that $Y$ is a connected topological space and $f:\left(Y, y_{0}\right) \rightarrow\left(X, x_{0}\right)$ is a continuous map of pointed topological spaces. If $\widetilde{x}_{0} \in \widetilde{X}$ such that $p\left(\widetilde{x}_{0}\right)=x_{0}$, then there exists at most one continuous map $\widetilde{f}:\left(Y, y_{0}\right) \rightarrow\left(\widetilde{X}, \widetilde{x}_{0}\right)$ such that $p \widetilde{f}=f$.

As a corollary of this lemma, the following can be restated

Corollary 1.3. Let $p: \widetilde{X} \rightarrow X$ be a covering map, $Y$ a connected topological space and let $f:\left(Y, y_{0}\right) \rightarrow\left(X, x_{0}\right)$ be a continuous map of pointed topological spaces. Let $\widetilde{x}_{0} \in \widetilde{X}$ such that $p\left(\widetilde{x}_{0}\right)=x_{0}$. If $g, h:\left(Y, y_{0}\right) \rightarrow\left(\widetilde{X}, \widetilde{x}_{0}\right)$ are the maps of pointed topological spaces such that $p g=p h=f$, then $g=h$.

Lemma 1.4.([9]) Let $p: \widetilde{X} \rightarrow X$ be a covering map, $Y$ a connected and locally path connected topological space and let $f:\left(Y, y_{0}\right) \rightarrow\left(X, x_{0}\right)$ be a continuous map of pointed spaces. Let $\widetilde{x}_{0} \in \widetilde{X}$ such that $p\left(\widetilde{x}_{0}\right)=x_{0}$. Then there exists a unique continuous map $\widetilde{f}:\left(Y, y_{0}\right) \rightarrow\left(\widetilde{X}, \widetilde{x}_{0}\right)$ such that $p \widetilde{f}=f$ if and only if $f_{\star}\left(\pi_{1}\left(Y, y_{0}\right)\right) \subseteq p_{\star}\left(\pi_{1}\left(\widetilde{X}, \widetilde{x}_{0}\right)\right.$ where $f_{\star}$ and $p_{\star}$ are respectively the induced morphisms from $f$ and $p$.

Corollay 1.5. Let $p: \widetilde{X} \rightarrow X$ be a covering map, $Y$ simply connected and locally path connected topological space and let $f:\left(Y, y_{0}\right) \rightarrow\left(X, x_{0}\right)$ be a continuous map of pointed spaces. If $\widetilde{x}_{0} \in \widetilde{X}$ such that $p\left(\widetilde{\sim}_{0}\right)=x_{0}$. Then there exists a unique continuous map $\widetilde{f}:\left(Y, y_{0}\right) \rightarrow\left(\widetilde{X}, \widetilde{x}_{0}\right)$ such that $p \widetilde{f}=f$.

Such a map $\tilde{f}$ is called a lifting of $f$. Hence if $Y$ is simply connected then $f$ lifts to each cover of $X$. 


\section{A Construction of Douady and Lazard}

The following construction is due to Douady and Lazard [5]. It is important because it gives a clear relation of covers and monodromy principles.

Let $G$ be a Lie group, with its group operation written additively (though $G$ need not be commutative). Let $W$ be an open neighbourhood of the identity element in $G$ and $i: W \rightarrow G$ the inclusion map. Let $F(W)$ be the free group on $W$ and let $N$ be the normal subgroup generated by the elements of $F(W)$ of the form $[u+v]-[u]-[v]$ for all $u, v \in W$ such that $u+v \in W$. Let $M(W)$ be the quotient group of $F(W)$ by $N$. Thus by the construction of $M(W)$ we have a map $\widetilde{\imath}: W \rightarrow M(W)$ and a morphism $p: M(W) \rightarrow G$ of groups with the property that if $f: W \rightarrow H$ is a local morphism of groups then there exists a unique group morphism $\phi: M(W) \rightarrow H$ such that $f=\phi \widetilde{i}$. Note that since $\tilde{p} \widetilde{\imath}=i$, then $\widetilde{\imath}$ is injective.

Up to now we do not have a topology on $M(W)$. Douady and Lazard [5] proved the following theorem

Theorem 2.1.([5]) Let $G$ be a Lie group and $W$ an open neighbourhood of the identity in $G$. Then there is a unique topology on the group $M(W)$ such that (i) left and right translations are homeomorphisms, and (ii) the function $\widetilde{\imath}: W \rightarrow M(W)$ is a homeomorphism to an open subspace. Further the morphism $p: M(W) \rightarrow G$ is a covering map on the underlying spaces.

Note that it is not claimed that $M(W)$ is, with this topology, a Lie group, and in general this will not be so, as the following remarks show.

We also have the following result.

Proposition 2.2. Let $G$ be a Lie group and $W$ an open neighbourhood of the identity in $G$. If $W$ is path connected then $M(W)$ is path connected.

The proof is analogous to that of Douady and Lazard, who give the 'connected' rather than 'path connected' case.

In [6] this theory was developed to the case where $G$ is a topological groupoid and written in a Lie groupoid version in [2] (see also [3]). In [2] the Lie case is stated in a form which includes the topological case, and this we assume here.

Brown and Mucuk in [2] proved the following result.

Theorem 2.3.(Corollary 2.6 in [2]) Suppose $G$ is a Lie group, $W$ is a path connected neighbourhood of the identity in $G$ such that $W$ generates $G$ and $i: W \rightarrow G$ is the inclusion. Let $p: M \rightarrow G$ be a morphism of groups and suppose given $\widetilde{\imath}: W \rightarrow M$ such that $\widetilde{\imath}(W)$ generates $M, p \widetilde{\imath}=i$ and $\widetilde{\imath}(u w)=(\widetilde{\imath} u)(\widetilde{\imath} w)$ whenever $u, w, u w$ belong to $W$. Then the group structure on $M$ gives $M$ the structure of Lie group such that $p: M \rightarrow G$ is a covering map and a morphism of Lie groups.

Consequently, we get in this way a monodromy principle for topological groups. 
Corollary 2.4. Let $G$ be a path connected Lie group and $W$ an open path connected neighbourhood of the identity in $G$ such that $W$ generates $G$. Then the group $M(W)$ can be given a structure of Lie group, and the morphism of groups $p: M(W) \rightarrow G$ is a covering map on the underlying spaces. If $f: W \rightarrow H$ is a local morphism of topological groups then there exists unique group morphism $\phi: M(W) \rightarrow H$ such that $f=\phi \widetilde{\imath}$.

\section{Universal Covers of Topological Modules and a Monodromy Principle}

We will apply these results to the case where $M$ is a topological left $R$-module.

A topological ring is a ring $R$ with a topology on the underlying set such that the structure maps $(x, y) \mapsto x+y, x \mapsto-x$ and $(x, y) \mapsto x y$ are all continuous.

A topological ring $R$ is called connected if the underlying space of $R$ is connected.

A morphism $f: R \rightarrow S$ of topological rings is a morphism of the underlying rings which is also continuous.

Definition 3.1. Let $R$ be a topological ring with identity $1_{R}$. A topological left $R$ module is an additive abelian topological group $M$ together with a continuous function (called an action of $R$ on $M) \delta: R \times M \rightarrow M$ defined by $(r, x) \mapsto r x$ such that

(i) $r(x+y)=r x+r y$

(ii) $(r+s) x=r x+s x$

(iii) $(r s) x=r(s x)$

(iv) $1_{R} x=x$

for $r, s \in R$ and $x, y \in M$.

As an example a topological vector space over $R$, the set of real numbers, is a topological left $R$-module.

Let $M$ and $N$ be topological left $R$-modules. A morphism of topological left $R$-modules is a group morphism $f: N \rightarrow M$ which is continuous and $f(r x)=r f(x)$ for $x \in N$ and $r \in R$. A morphism $f: N \rightarrow M$ of topological left $R$-modules is called cover if $f$ is a covering morphism on the underlying topologies.

The following result is well known in the theory of covering spaces (see for example Chevalley's book [4]) which can be proved using Corollary 1.3.

Proposition 3.2. Let $G$ be a path connected additive topological group with identity element 0 and $\widetilde{G}$ a simply connected topological space. Let $p: \widetilde{G} \rightarrow G$ be a covering map on the underlying spaces and $\widetilde{0}$ an element of $\widetilde{G}$ such that $p(\widetilde{0})=0$. Then there exists a group structure on $\widetilde{G}$ with identity element $\widetilde{0}$ such that $\widetilde{G}$ is a topological group with this group structure and $p: \widetilde{G} \rightarrow G$ is a morphism of topological groups.

A similar result for topological rings rather than topological groups is given in [8].

Remark 3.3. We note that using Lemma 1.4 it can be proved that Proposition 3.2 is still true in the case where $\widetilde{G}$ is connected rather then simply connected.

We now prove a similar result for topological left $R$-modules. 
Proposition 3.4. Let $R$ be a simply connected topological ring with identity $1_{R}$. Let $M$ be a path connected topological left $R$-module such that 0 is the identity element of the additive group structure of $M$ and $\widetilde{M}$ a connected topological space. Let $p: \widetilde{M} \rightarrow M$ be a covering map on the underlying spaces and $\widetilde{0}$ an element of $\widetilde{M}$ such that $p(\widetilde{0})=0$. Then there exists a topological left $R$-module structure on $\widetilde{M}$ such that $\widetilde{0}$ is the identity element of the additive group structure of $\widetilde{M}$ and $p$ is a morphism of topological left R-modules.

Proof. Let $M$ be a path connected topological left $R$-module given by an action $\delta: R \times M \rightarrow M$ and 0 the identity element of the additive group structure of $M$. Let $\widetilde{0}$ be an element of $\widetilde{M}$ such that $p(\widetilde{0})=0$. Since $\widetilde{M}$ is connected by Proposition 3.2 there is a group structure on $\widetilde{M}$ such that $\widetilde{M}$ is a topological group, $\widetilde{0}$ is an identity element of $\widetilde{M}$ and $p: \widetilde{M} \rightarrow M$ is a morphism of topological groups.

Consider the map of pointed topological spaces $f: R \times \widetilde{M},\left(1_{R}, \widetilde{0}\right) \rightarrow M, 0$ defined by $f(r, \widetilde{x})=r p(\widetilde{x})$. Since $f=\delta(1 \times p)$ as a composite of the continuous maps is continuous and since $R$ is simply connected we have that

$$
f_{\star}\left(\pi_{1}\left(R \times \widetilde{M},\left(1_{R}, \widetilde{0}\right)\right) \subseteq p_{\star}\left(\pi_{1}(\widetilde{M}, \widetilde{0})\right),\right.
$$

because for a path $\widetilde{a}$ at $\widetilde{0}$ in $\widetilde{M}$ and a path $r$ in $R$ at $1_{R}$ we have

$$
\begin{aligned}
f_{*}([r, \widetilde{a}]) & =\delta_{*}(1 \times p)_{*}([r, \widetilde{a}]) \\
& =\delta_{*}([r, p(\widetilde{a})]) \\
& =([r p(\widetilde{a})]) \\
& =[r][p(\widetilde{a})]
\end{aligned}
$$

and since $R$ is simply connected, $[r]=[1]$ and so that

$$
f_{*}([r, \widetilde{a}])=[p(\widetilde{a})] \in p_{*}\left(\pi_{1}(\widetilde{M}, \widetilde{0})\right) .
$$

Hence by Lemma 1.4 there exists a unique continuous map

$$
\widetilde{\delta}: R \times \widetilde{M},\left(1_{R}, \widetilde{0}\right) \rightarrow \widetilde{M}, \widetilde{0}
$$

such that $p \widetilde{\delta}=f$.

We write $r \widetilde{x}$ for $\widetilde{\delta}(r, \widetilde{x})$. Here note that $1_{R} \widetilde{0}=\widetilde{0}$. We now prove that the following properties are satisfied:

(i) $r(\widetilde{x}+\widetilde{y})=r \widetilde{x}+r \widetilde{y}$

(ii) $(r+s) \widetilde{x}=r \widetilde{x}+s \widetilde{x}$

(iii) $(r s) \widetilde{x}=r(s \widetilde{x})$

(iv) $1_{R} \widetilde{x}=\widetilde{x}$

for $r, s \in R$ and $\widetilde{x}, \widetilde{y} \in M$.

To prove (i) consider the map of pointed spaces

$$
f: R \times \widetilde{M} \times \widetilde{M},\left(1_{R}, \widetilde{0}, \widetilde{0}\right) \rightarrow M, 0
$$


defined by $f(r, \widetilde{x}, \widetilde{y})=r(\widetilde{x}+\widetilde{y})$ and define the functions of pointed spaces

$$
g, h: R \times \widetilde{M} \times \widetilde{M},\left(1_{R}, \widetilde{0}, \widetilde{0}\right) \rightarrow \widetilde{M}, \widetilde{0}
$$

by $g(r, \widetilde{x}, \widetilde{y})=r(\widetilde{x}+\widetilde{y})$ and $h(r, \widetilde{x}, \widetilde{y})=r \widetilde{x}+r \widetilde{y}$. Note that $p g=p h$ and since $1_{R} \widetilde{0}=\widetilde{0}$ we have that $g\left(1_{R}, \widetilde{0}, \widetilde{0}\right)=h\left(1_{R}, \widetilde{0}, \widetilde{0}\right)$. So by Corollary 1.3 , we have that $g=h$. That means for $r \in R$ and $\widetilde{x}, \widetilde{y} \in M$ we have $r(\widetilde{x}+\widetilde{y})=r \widetilde{x}+r \widetilde{y}$ which proves that the condition (i) is satisfied.

The proofs of (ii) and (iii) are similar. So it is sufficient to prove the condition (iv). For this consider the map of pointed spaces $f:\left\{1_{R}\right\} \times \widetilde{M},\left(1_{R}, \widetilde{0}\right) \rightarrow M, 0$ defined by $f\left(1_{R}, \widetilde{x}\right)=1_{R} p(\widetilde{x})=p(\widetilde{x})$. Define the maps of pointed spaces $g, h:\left\{1_{R}\right\} \times \widetilde{M},\left(1_{R}, \widetilde{0}\right) \rightarrow$ $\widetilde{M}, \widetilde{0}$ to be $g\left(1_{R}, \widetilde{x}\right)=1_{R} \widetilde{x}$ and $h\left(1_{R}, \widetilde{x}\right)=\widetilde{x}$. Then $p g=p h=f$. So by Corollary 1.3 we have that $g=h$. That means $1_{R} \widetilde{x}=\widetilde{x}$, for $\widetilde{x} \in \widetilde{M}$, which proves (iv).

Let $M$ be a topological left $R$-module and $W$ an open neighborhood of the identity element 0 of $M$. We say $W$ generates $M$ additively if each element of $M$ can be written as an additive of some elements of $W$.

Let $M$ be a topological left $R$-module and $W$ an open neighbourhood of the identity element 0 of additive group structure of $M$. We now apply the construction $M(W)$ of section 2 to the additive group of $M$ and $W$.

Proposition 3.5. Let $R$ be a simply connected topological ring with identity $1_{R}$. Let $M$ be a topological left $R$-module and $W$ an open, path connected neighbourhood of the identity element 0 of the additive group of $M$ such that $W$ generates $M$ additively. Then $M(W)$ becomes a topological left $R$-module such that the map $p: M(W) \rightarrow M$ is a covering morphism of topological left $R$-modules.

Proof. Since $W$ is path connected and generates $M$ additively, the topological left $R$-module $M$ is path connected and by Proposition 2.2 when $W$ is path connected, $M(W)$ is also path connected. By Theorem 2.1, $M(W)$ has a topology such that the map $p: M(W) \rightarrow M$ is a connected cover on the underlying spaces. So by Proposition 3.4 $M(W)$ becomes a topological left $R$-module such that $p: M(W) \rightarrow M$ is a morphism of topological left $R$-modules.

Definition 3.6. Let $M$ and $N$ be topological left $R$-modules and $W$ an open neighbourhood of the identity element 0 of the additive group of $M$. A continuous map $\phi: W \rightarrow N$ is called a local morphism of topological left $R$-modules if $\phi(u+v)=\phi(u)+\phi(v)$ and $\phi(x u)=x \phi(u)$ for $u, v \in W$ and $x \in R$ such that $u+v$ and $x u$ are in $W$.

Proposition 3.7. Let $M$ and $\widetilde{M}$ be topological left $R$-modules and $q: \widetilde{M} \rightarrow M a$ covering morphism of topological left $R$-modules. Let $W$ be an open, path connected neighbourhood of the identity element 0 in $M$ such that $W+W$ and $R W$ are contained in a liftable neighbourhood $V$ of 0 in $M$. Then the inclusion map $i: W \rightarrow M$ lifts to a local morphism $\hat{\imath}: W \rightarrow \widetilde{M}$ of topological left $R$-modules. 
Proof. Since $V$ lifts to $\widetilde{M}$ then $W$ lifts to $\widetilde{M}$ by $\hat{\imath}: W \rightarrow \widetilde{M}$. We now prove that $\hat{\imath}$ is a local morphism of topological left $R$-modules. We know by the lifting theorem that $\hat{\imath}: W \rightarrow \widetilde{M}$ is continuous. We have prove that $\hat{\imath}: W \rightarrow \widetilde{M}$ is a morphism of topological left $R$-modules. Let $u, v \in W$ and $x \in R$ such that $u+v$ and $x u$ are in $W$. Let $a$ and $b$ be the paths from $u$ and $v$ to 0 in $W$ respectively. Let $c=a+b$ so that $c$ is a path from $u+v$ to 0 . Since $W+W \subseteq V$, the path $c$ is in $V$. So the paths $a, b$ and $c$ lift to $\widetilde{M}$. Let $\widetilde{a}, \widetilde{b}$ and $\widetilde{c}$ be the liftings of $a, b$ and $c$ in $\widetilde{M}$ respectively. Then we have

$$
q(\widetilde{c})=c=a+b=q(\widetilde{a})+q(\widetilde{b}) .
$$

Because $q$ is a morphism of modules,

$$
q(\widetilde{a}+\widetilde{b})=q(\widetilde{a})+q(\widetilde{b}) .
$$

Since $\widetilde{c}, \widetilde{a}+\widetilde{b}$ end at $\widetilde{0} \in \widetilde{M}$,

$$
\widetilde{c}=\widetilde{a}+\widetilde{b}
$$

by unique path lifting. On evaluating these paths at $0 \in I$ we have

$$
\hat{\imath}(u+v)=\hat{\imath}(u)+\hat{\imath}(v) .
$$

Let the path $x a$ be defined by

$$
(x a)(t)=x(a(t)), \quad t \in I .
$$

Since $R W \subseteq V$, the path $x a$ is a path in $V$ from $x u$ to 0 . Let $\widetilde{x a}$ be the lifting of the path $x a$ by $\hat{\imath}$. So

$$
q(\widetilde{x a})=x a .
$$

Since $q$ is a morphism of topological modules

$$
q(x \widetilde{a})=x q(\widetilde{a})=x a .
$$

By the unique path lifting

$$
\widetilde{x a}=x \widetilde{a} .
$$

On evaluating these paths at $0 \in I$ we have

$$
\hat{\imath}(x u)=x \hat{\imath}(u)
$$

Hence $\hat{\imath}: W \rightarrow \widetilde{M}$ is a local morphism of topological left $R$-modules.

Theorem 3.8. Let $R$ be a simply connected topological ring with identity $1_{R}$ and $M$ a topological left $R$-module whose underlying space is path connected and admits a simply connected cover. Let $W$ be an open, path connected neighbourhood of the identity element 0 in $M$ such that $W+W$ and $R W$ are contained in a liftable neighbourhood $V$ of 0 . Then the covering map $p: M(W) \rightarrow M$ is a universal cover of topological left $R$-modules. 
Proof. By Proposition 3.5, $M(W)$ is a topological left $R$-module and $p: M(W) \rightarrow M$ is a covering morphism of topological left $R$-modules. To prove that the map $p: M(W) \rightarrow$ $M$ is universal we have to verify the universal property, that means $M(W)$ covers every cover of $M$. For let $q: \widetilde{M} \rightarrow M$ be a cover such that $\widetilde{M}$ is path connected. Choose $\widetilde{0} \in \widetilde{M}$ such that $q(\widetilde{0})=0$. Then by Proposition $3.4, \widetilde{0}$ defines a topological left $R$ module structure on $\widetilde{M}$ such that $\widetilde{0}$ is the identity element in $\widetilde{M}$ and $q$ is a morphism of topological left $R$-modules. By Proposition 3.7 the inclusion map $i: W \rightarrow M$ lifts to a local morphism of topological $R$-modules $\hat{\imath}: W \rightarrow \widetilde{M}$. Then $\hat{\imath}$ extends to a morphism $f: F(W) \rightarrow \widetilde{M}$. For $(u, v) \in W \times W$ such that $u+v \in W$ we have

$$
\begin{aligned}
f([u+v]-[u]-[v]) & =f([u+v])-f([u])-f([v]) \\
& =\hat{\imath}(u+v)-\hat{\imath}(u)-\hat{\imath}(v) \\
& =\widetilde{0} .
\end{aligned}
$$

Hence the morphism $f: F(W) \rightarrow \widetilde{M}$ determines a morphism $\phi: M(W) \rightarrow \widetilde{M}$ such that $\phi \theta=f$, where $\theta: F(W) \rightarrow M(W)$ is the quotient map. Then $\phi$ becomes a covering map. For this it is sufficient to prove that $\phi$ is continuous. By the construction of $M(W)$ we have an inclusion $\widetilde{\imath}: W \rightarrow M(W)$. Denote the image of $W$ under this inclusion by $\widetilde{W}$. Then $\widetilde{W}$ is open in $M(W), \widetilde{W}$ generates $M(W)$ and the morphism $\phi: M(W) \rightarrow \widetilde{M}$ is continuous on $\widetilde{W}$. Thus the continuity of $\phi$ elsewhere follows.

We now give the following theorem as a monodromy principle for topological left $R$-modules.

Theorem 3.9. (Monodromy Principle) Let $R$ be a simply connected topological ring and $M$ a topological left $R$-module whose underlying topology is path connected and admits a simply connected cover. Let $W$ be an open, path connected neighbourhood of the identity element 0 in $M$ such that $W+W$ and $R W$ are contained in a liftable neighbourhood $V$ of 0 . Then there exists a local morphism $\widetilde{\imath}: W \rightarrow M(W)$ of topological left $R$-modules such that if $f: W \rightarrow N$ is a local morphism from $M$ to a topological left $R$-module $N$ then there is a unique morphism $\phi: M(W) \rightarrow N$ of topological left $R$-modules such that $f=\phi \widetilde{\imath}$. Further the projection morphism $p: M(W) \rightarrow M$ is a universal cover on topological left $R$-modules.

Proof. By the construction of $M(W)$ there is an inclusion map $\widetilde{\imath}: W \rightarrow M(W)$ and by Proposition 3.5 $M(W)$ is a topological left $R$-module. If $f: W \rightarrow N$ is a local morphism of topological left $R$-modules this gives us a unique morphism $\phi: M(W) \rightarrow N$ of topological left $R$-modules such that $\phi \widetilde{\imath}=f$. Since $\phi$ is continuous on $\widetilde{W}=\widetilde{\imath}(W)$ which is open in $M(W)$ and generates $M(W)$, the continuity of $\phi$ follows. Further by Theorem 3.8 the projection morphism $p: M(W) \rightarrow M$ is a universal cover. 


\section{Acknowledgement}

We would like to thank Prof. R. Brown for introducing this area and to the referee for helpful comments.

\section{References}

[1] Brown, R. and Mucuk, O., Covering groups of non-connected topological groups revisited, Math. Proc. Camb. Phill. Soc. 115(1994), 97-110.

[2] Brown, R. and Mucuk, O., Monodromy groupoid of a Lie Groupoid, Cah. Top. Géom. Diff. Cat. 36(1995), 345-370.

[3] Brown, R. and Mucuk, O., Foliations, locally lie groupoids and Holonomy, Cah. Top. Géom. Diff. Cat. 37(1996), 61-67.

[4] Chevalley, C., Theory of Lie Groups, Princeton University Press, 1946.

[5] Douady L. and Lazard, M., Espaces fibrés en algèbres de Lie et en groupes, Invent. Math. 1(1966), 133-151.

[6] Mucuk, O., Covering groups of non-connected topological groups and the monodromy groupoid of a topological groupoid, Ph.D. Thesis, University of Wales, 1993.

[7] Mucuk O., Coverings and ring-groupoids, Geor. Math. J. 5(1998), 475-482.

[8] Mucuk, O., Özdemir, M., A monodromy principle for the universal coverings of topological rings., Ind. Jour. Pure and App. Math. 31(2000), 1531-1535.

[9] Rotman, J. J., An Introduction to Algebraic Topology, Graduate Texts in Mathematics 119(1988), Springer-Verlag, Newyork.

[10] Taylor, R. L., Covering groups of non-connected topological groups, Proc. Amer. Math. Soc. 5(1954), 753-768.

Department of Mathematics, Erciyes University, Kayseri 38039, Turkey.

E-mail: mucuk@erciyes.edu.tr 